\title{
(Our response to) COVID-19: In science we trust
}

\author{
Sebastiano Filetti ${ }^{1}$ \\ Published online: 21 January 2021 \\ (c) The Author(s), under exclusive licence to Springer Science+Business Media, LLC part of Springer Nature 2021
}

To the readers of Endocrine,

2020 has drawn to a close, and an end to the pandemic that has made this year so disastrous is now in sight, thanks to an unprecedented scientific achievement: in the 10 months that have passed since the first cases of "atypical pneumonia" were reported in China, the molecular structure of the virus that causes COVID-19 has been identified, and vaccines capable of defending us against that virus have already been produced, and are now being administered throughout the world. The possibility that we will soon be able to recover some sense of normalcy is exhilarating, the desire to put this annus horribilis behind us overwhelming.

But before we race ahead, it is imperative that we look back and reflect on what exactly has happened. During the final months of 2019, yet another zoonotic virus was making the leap from animals to human hosts. Unlike many of its predecessors, this one spread like wildfire and quickly began to cause suffering with almost 2 million deaths, leaving in its wake grieving disoriented communities, shattered economies, healthcare systems on the verge of collapse, and a pervasive sense of uncertainty.

And yet, 2020 also brought with it hard but invaluable lessons that we can not afford to forget:

First, our planet is crisscrossed with essential networks that ensure almost real-time global distribution and redistribution of food, livestock, raw materials, manufactured goods, and last but not least human beings. Science has shown us unequivocally how these networks distribute viruses and other pathogens as well. It is thus essential that our concept of "health" and "healthcare" needs to be broadened, made more global. It would be short-sited [myopic] for any country to believe that health can be achieved or maintained in national terms alone.

Sebastiano Filetti

editor.endocrine@gmail.com

School of Health, UnitelmaSapienza, Rome, Italy
Second, the lockdowns imposed on societies to slow the alarming spread of this previously unknown disease have had equally disruptive effects. The restrictions have had a dismaying impact on virtually all the activities of our daily life: shopping, working, learning, teaching, socializing, commuting, traveling, healthcare activities (from routine prevention to urgent diagnostic procedures and treatment), etc. have all been appreciably restricted [o disrupted]. And yet, remote alternatives and work-arounds are being discovered, distributed, improved, mastered, and updated with remarkable speed, and once again, these solutions have been provided by the fields of science and technology.

The world's remarkable response to the COVID-19 pandemic is rooted in active and often innovative forms of collaboration and exchange, among scientists across the world, and in the combined power of public and private investments.

The field of endocrinology, like other disciplines, has been strongly impacted by this pandemic. Patients with acute and chronic endocrine disease have suddenly been faced with delays or suspension of care and medical advice (sometimes self-imposed for fear of contagion) they once considered essential (e.g., routine follow-up visits, treatments, surgery, diagnostic procedures). But COVID-19 also has numerous direct effects on the endocrine system that have been and continue to be described [1]. Endocrine has been at the forefront in documenting and promoting awareness of these aspects of the disease, as reflected by our publication of the ESE statement outlining the effect of COVID-19 on the endocrine system [1] and editorials calling attention to the evolving changes, the emerging challenges, and new scenarios introduced by COVID-19 [2, 3].

Since the April 2020 issue, Endocrine has published 29 articles dealing with the endocrine effects and implications of infection with SARS-CoV-2. Springer has already collected these papers and published them on the Endocrine web site as a single open-access portfolio readily available to all readers. 
The year 2020 has witnessed a $45 \%$ increase in submissions compared to 2019, and Endocrine's impact is steadily growing, with roughly one-third of the readers who access the journal hailing from North or South America (31\%), Asia and the Pacific (32\%), or Europe (28\%). We look forward to increasing our visibility in the Middle East and Africa in 2021. The growing number of papers submitted for publication has put an undeniable strain on our review process. We have made every attempt to expedite the publication of COVID-19-related papers without sacrificing the quality standards that are our hallmark. Despite these efforts, delays in some of our responses to authors have occurred.

What are our plans for the next 12 months? First and foremost, an expanded editorial board that can accelerate and improve our ability to provide readers with high-quality, original, and innovative contents dealing with the management of endocrine diseases in the COVID-19 and post-COVID-19 era. We intend to focus on novel, less frequently examined topics such as Information Technology applications in the field of endocrinology (IT), PatientCentered Outcomes Research, and the effects of environmental and climate changes on endocrine diseases. I am also excited to announce that in March, Endocrine will publish an entire issue devoted specifically to rare endocrine conditions, with the collaboration of numerous Endo-ERN (European Reference Network on Rare Endocrine Conditions) experts and co-edited by Alberto Pereira and Olaf Hiort. With this initiative, we hope to draw attention to the need for concrete actions to support patients with these diseases, whose suffering has been markedly exacerbated by the pandemic.

Before I close, let me also thank my colleagues on the Editorial Board for the heavy workload they have shouldered over the past year, Bex Chang, the journal's Publishing Editor, and Aiswarya Satheesan and Prathap Panneerselvam, the journal's editorial assistant and production editor, respectively. Their commitment and generosity over the past 12 months have been extraordinary and remarkable. And last but not least, you, our readers and contributors: your manuscripts are the beating heart of the journal, and we are counting on you to help us provide the international community of endocrinologic researchers and clinicians with reliable, thought-provoking guidance for 2021 - the science in which all of us must continue to place our trust.

Best wishes to all.

Sebastiano Filetti, MD

\section{Compliance with ethical standards}

Conflict of interest The authors declare that they have no conflict of interest.

Publisher's note Springer Nature remains neutral with regard to jurisdictional claims in published maps and institutional affiliations.

\section{References}

1. M. Puig-Domingo, M. Marazuela, A. Giustina, COVID-19 and endocrine diseases. A statement from the European Society of Endocrinology. Endocrine 68, 2-5 (2020). https://doi.org/10.1007/ s12020-020-02294-5

2. S. Filetti, The COVID-19 pandemic requires a unified global response. Endocrine 68, 1 (2020). https://doi.org/10.1007/s12020020-02293-6

3. S. Filetti, A journey through and beyond a "perfect storm": the COVID-19 pandemic. Endocrine 68, 249-250 (2020). https://doi. org/10.1007/s12020-020-02336-y 